\title{
Descriptive study of X-class flares released in the year 2014, during the double peak of SC-24
}

\author{
Ahmed A. Hady ${ }^{1}$, Marwa H. Mostafa ${ }^{1}$ and Susan W. Samwel ${ }^{2}$ \\ ${ }^{1}$ Department of Astronomy \& Space and Meteorology, Faculty of Science, Cairo University \\ Giza, Egypt \\ email: aahady@sci.cu.edu.eg \\ ${ }^{2}$ National Research Institute of Astronomy and Geophysics (NRIAG) \\ Helwan, Cairo, Egypt
}

\begin{abstract}
During the declining phase of the Solar cycle 24, a new peak appeared on January 7 , 2014. The release of $\mathrm{x}$-class flares, with the high energetic particles, were found to be more intense than that occurred during the main peak of the same cycle. Few X-class flares were released, lately, during the year 2014. We note that during the last 5 solar cycles, a new peak has appeared, releasing high energetic particles and X-class solar flares, which are called the secondary peak or the double peak of solar cycle. The aim of this descriptive study is to follow the morphological and magnetic changes of the active region before, during, and after the production of X-class flares according to data analysis. Furthermore, the causes of the release of such eruptive storms have been discussed for the period, year 2014, during the double peak of the solar cycle 24 .
\end{abstract}

Keywords. Solar cycle 24, solar activity, X-class flares, solar energetic particles.

\section{Introduction}

The complex dynamics of magnetic fields play a key role in the solar activity (Parker, 2001). The present solar cycles 24 , and the previous one, solar cycle 23 , are the weakest cycles observed in the last 200 years (Hady, 2013) and (Hady, 2014). The solar cycle 23 began in May 1996 with smoothed sunspot number, and peaked in April 2001. However, a sudden increase of activities occurred during the period of so-called Halloween storms, in October-November 2003. Similar storms occurred one year later, during the period from October 3 to November 13, 2004 (Hady, 2009). The underlying physics of the solar cycles 23 and 24 is not fully understood till the present time (Kane, 2008) and (Solheim et al., 2012). The aim of the present work is to study the eruptive solar events during 2014 especially that released x-class flares. Descriptive and analytical studies are given to follow the morphological and magnetic changes of the active region before, during, and after the production of X-class flares during the double peak of the solar cycle 24 .

\section{Data and Observations}

The dataset considered in the present study is based on the eruptive solar events associated with soft X-ray flares of X class (peak flux $\sim 10^{-4} \mathrm{Wm}^{-2}$ ) during 2014. Nine events of x-class flares were observed and examined in this paper. The daily solar data of the solar events under study are represented in table 1 . The first column represents the date of the events in Year, month, and day. The second column represents the $10.7 \mathrm{~cm}(2800$ $\mathrm{MHz}$ ) full Sun background radio flux on the date indicated in units of $10^{-22} \mathrm{Wm}^{-2} \mathrm{~Hz}^{-1}$. The third column shows the Space Environment Services Center (SESC) sunspot number 
Table 1. Daily solar data for x-class flare days occurring through the year 2014

\begin{tabular}{|c|c|c|c|c|c|c|c|c|c|c|c|c|}
\hline \multirow[t]{3}{*}{ Date } & \multirow{3}{*}{$\begin{array}{l}\text { Radio } \\
\text { Flux } \\
10.7 \\
\mathrm{~cm}\end{array}$} & \multirow{3}{*}{$\begin{array}{l}\text { SSESC } \\
\text { Sunspot } \\
\text { Num- } \\
\text { ber }\end{array}$} & \multirow{3}{*}{$\begin{array}{l}\text { Sunspot } \\
\text { Area } \\
10^{-6} \text { Hemis. }\end{array}$} & \multirow{3}{*}{$\begin{array}{l}\text { Sunspot } \\
\text { Region }\end{array}$} & \multirow{3}{*}{$\begin{array}{l}\text { Flare } \\
\text { inten- } \\
\text { sity }\end{array}$} & \multicolumn{7}{|c|}{ Flares } \\
\hline & & & & & & \multicolumn{3}{|c|}{ X-Ray } & \multicolumn{4}{|c|}{ Optical } \\
\hline & & & & & & $\mathrm{C}$ & M & $\mathrm{X}$ & $\mathrm{S}$ & 1 & 2 & 3 \\
\hline 20140107 & 237 & 196 & 1850 & AR1944 & $\mathrm{X} 1.2$ & 8 & 2 & 1 & 13 & 1 & 2 & 0 \\
\hline 20140225 & 174 & 157 & 910 & AR1990 & $\mathrm{X} 4.9$ & 4 & 0 & 1 & 11 & 1 & 1 & 0 \\
\hline 20140329 & 143 & 132 & 470 & AR2017 & $\mathrm{X} 1.0$ & 14 & 0 & 1 & 15 & 1 & 1 & 0 \\
\hline 20140425 & 125 & 73 & 390 & AR2046 & $\mathrm{X} 1.3$ & 4 & 0 & 1 & 1 & 0 & 0 & 0 \\
\hline 20140610 & 166 & 149 & 1240 & AR2087 & $\mathrm{X} 2.2$ & 6 & 0 & 2 & 6 & 1 & 0 & 0 \\
\hline 20140611 & 168 & 176 & 1490 & AR2087 & $\mathrm{X} 1.3$ & 10 & 3 & 1 & 19 & 0 & 2 & 0 \\
\hline 20140910 & 160 & 161 & 1070 & AR2158 & X1.6 & 1 & 0 & 1 & 12 & 0 & 1 & 0 \\
\hline 20141019 & 173 & 86 & 1850 & AR2192 & $\mathrm{X} 1.0$ & 7 & 0 & 1 & 10 & 0 & 0 & 1 \\
\hline 20141022 & 216 & 123 & 3120 & AR2192 & $\mathrm{X} 1.3$ & 4 & 3 & 1 & 9 & 0 & 1 & 0 \\
\hline 20141024 & 218 & 147 & 2820 & AR2192 & X 3.0 & 5 & 1 & 1 & 10 & 0 & 0 & 1 \\
\hline 20141025 & 219 & 115 & 2810 & AR2192 & $\mathrm{X} 1.0$ & 7 & 0 & 1 & 7 & 1 & 0 & 1 \\
\hline 20141026 & 217 & 138 & 3020 & AR2192 & $\mathrm{X} 2.0$ & 10 & 4 & 1 & 9 & 0 & 1 & 0 \\
\hline 20141027 & 188 & 120 & 2530 & AR2192 & $\mathrm{X} 2.0$ & 6 & 5 & 1 & 1 & 1 & 2 & 1 \\
\hline 20141107 & 146 & 96 & 510 & AR2205 & $\mathrm{X} 1.0$ & 6 & 3 & 1 & 7 & 0 & 1 & 1 \\
\hline 20141220 & 203 & 120 & 1750 & AR 2242 & $\mathrm{X} 2.0$ & 5 & 0 & 1 & 12 & 1 & 0 & 1 \\
\hline
\end{tabular}

for the date indicated as computed according to the Wolf Sunspot number equation. The $4^{\text {th }}$ column represents the sunspot area. The $5^{\text {th }}$ column represents the sunspot region number. The $6^{\text {th }}$ column shows the X-class flares intensity. The $7^{\text {th }}$ column is divided into two columns, representing the X-ray flare and optical flare intensity respectively. The data obtained from the Daily Solar Data were given from U.S. Dept. of Commerce, NOAA, and Space Weather Prediction Center:

http://legacy-www.swpc.noaa.gov/weekly/index.html, ftp://ftp.swpc.noaa.gov/pub/ indices/old_indices/2014_DSD.txt

In the present study, 6 daily solar parameters were analyzed, namely, $10.7 \mathrm{~cm}$ radio flux, sunspot number, sunspot area, daily average background x-ray flux, X-ray flare intensity and optical flare intensity. A MATHEMATICA program was constructed to analyze the six solar indices mentioned above. The program is based on the ascending cumulative frequency table. It is found that the effect of the eruptive X-class flares was clearly appeared, for the majority of the events understudy, only on three parameters; X-ray Background Flux, X-ray Flares intensity and Optical Flares intensity. The changes started, at least, one day before the release of X-class flares. This means that we can have a one day forecast before the X-class flare release using this simplified technique. This result is consistent with the finding of (Samwel et al., 2006) that the x-ray flares showed significant changes during the great solar proton events of the solar cycle 23 . The highly variation of sunspot number and area during the x-class flare release took place early before the occurrence the events. These variations disappeared in our accumulation curve analysis. As an example, the time profiles of the three solar indices; X-ray Background Flux, X-ray Flares intensity and Optical Flares intensity, for the event which took place on the $7^{\text {th }}$ of January 2014 are represented in Fig. 1(a, b, and c) respectively during and after the release of the eruptive flares. 

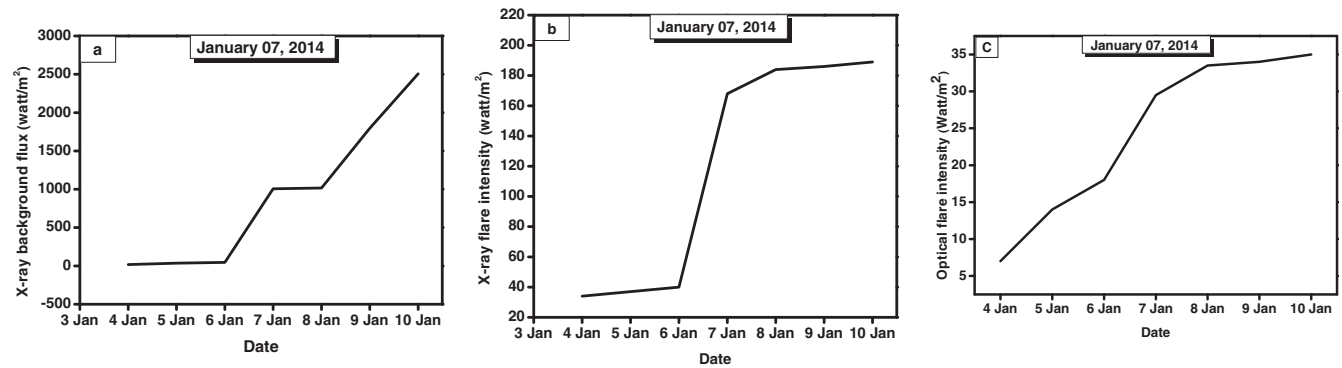

Figure 1. The time profiles of: a) x-ray background flux, b) x-ray flare intensity, and c) optical flare intensity of the event occurred on $7^{t h}$ of January 2014

\section{Conclusions}

The six solar parameters were analyzed. Sunspot active region AR1990 (formerly AR1967) returned to the earth side of the sun on Feb. $25^{t h}, 2014$, and promptly erupted, producing an X4. 9-class solar flare. This is the strongest flare of the year, but the giant Sunspot AR2192 is the biggest sunspot in nearly 25 years. It covers 2750 millionths of the solar disk. The changes of the temporal behavior of the sunspot number and area during the x-class flare release happened earlier before the occurrence of the events. This means that these two parameters can be used for a short time forecast, one day earlier before the occurrence of the x-class Flares. The optical flares intensity parameter shows no change in all events under study except in the event which took place in April 25, 2014. At this event, a considerable change was noted, two days after the occurrence of the flare event, attributed to the wavelength of the Optical Flares intensity. The effects of the eruptive X-class flares clearly appeared on three parameters: X-ray Background Flux, X-ray Flares intensity and Optical Flares intensity. The cause of this phenomenon is related to the changes of the area of the active regions which releases these events. The temporal behavior of the 6 parameters shows changes due to some degree, to the flare releasing, However, the time profile of the Radio Flux, Sunspot Numbers and Sunspot Area shows trivial changes compared to the x-ray background flux, X-ray Flare intensity and optical flare intensity.

\section{References}

Hady, A., A. 2009, JASTP, 71, 1716

Hady, A. A. 2013, Journal of Advanced Research, 4, 214

Hady, A. A. 2013, JGRE, 2, 157

Kane, R. P. 2008, Ann. Geophys., 26, 3339

Parker, E. N. 2001, Chinese Journal of Astronomy \& Astrophysics, 1, 124

Solheim, J.-E. \& Stordahl, K., Humlum,O. 2012, Journal of Atmospheric and Solar-Terrestrial Physics, 80, 284

Samwel S. W., Hady, A. A., Ibrahim, M., \& and Hanna, Y. S. 2006, Proceeding of IAU233 symposium, "Solar Activity and its Magnetic Origin, 287 